Herbert, D., Elsworth, R. \& Telling, R. C. (1956). J. gen. Microbiol. 14, 601-622

\title{
The Continuous Culture of Bacteria; a Theoretical and Experimental Study
}

\author{
By D. HERBERT, R. ELSWORTH AND R. C. TELLING \\ Microbiological Research Department (Ministry of Supply), Porton, Wiltshire
}

\begin{abstract}
SUMMARY: A theoretical treatment of continuous culture is given, which allows quantitative prediction of the steady-state concentrations of bacteria and substrate in the culture, and how these may be expected to vary with change of medium, concentration and flow-rate. The layout and operation of a small pilot plant for the continuous culture of bacteria are described. This plant has been operated continuously for periods of up to 4 months without breakdown or contamination of the culture. No alterations in the properties of the organisms studied have occurred during such periods of continuous culture. Results are given of a series of experiments on the continuous culture of Aerobacter cloacae in a chemically defined medium, designed to allow quantitative comparison with the results predicted by the theory. The relative advantages of batch and continuous culture as production processes are discussed, and it is concluded that continuous culture may usually be expected to show a five to tenfold increase in output as compared with a batch process.
\end{abstract}

The continuous culture of micro-organisms is a technique of increasing importance in microbiology. The essential feature of this technique is that microbial growth in a continuous culture takes place under steady-state conditions; that is, growth occurs at a constant rate and in a constant environment. Such factors as $\mathrm{pH}$ value, concentrations of nutrients, metabolic products and oxygen, which inevitably change during the 'growth cycle' of a batch culture, are all maintained constant in a continuous culture; moreover, they may be independently controlled by the experimenter. These features of the continuous culture technique make it a valuable research tool, while it offers many advantages, in the form of more economical production techniques, to the. industrial microbiologist. Nevertheless, the technique has so far been comparatively little used. (The review of Novick (1955) lists nearly all the work on the subject that has yet appeared.) The reasons for this relative neglect are, we believe, twofold.

The first reason is the lack of a generally accepted theoretical background. Continuous culture presents theoretical problems of an essentially kinetic nature which must be solved before the technique can be intelligently applied. The basis of a correct theoretical treatment has, in fact, already been laid in important papers by Monod (1950) and Novick \& Szilard (1950), but later writers on the subject (Golle, 1953; Finn \& Wilson, 1954; Northrop, 1954) have either disagreed with, or failed to understand, this work; at least their theoretical treatments are quite different (and in our view erroneous). This may have been because the earlier writers presented little experimental data in support of their theoretical conclusions. One object of the present work was to obtain quantitative data to test the general validity of Monod's theory. 
A second reason for the neglect of continuous culture techniques, in particular by industrial microbiologists, is the apparently widespread belief that they are so difficult as to be impracticable. Difficulty in maintaining sterility during long runs and the probability of mutations are two objections which have frequently been raised (e.g. Warner, Cook \& Train, 1954a, b). These objections, which have been answered by Dawson \& Pirt (1954), are based solely on conjecture since no serious attempts have hitherto been made (in Great Britain at least) to apply continuous techniques in industrial microbiology. Our own experience shows that these purely practical difficulties have been greatly exaggerated.

The present paper attempts to deal with both of the aspects of continuous culture mentioned above. In the first part a mathematical theory of continuous culture is presented; the second part describes the operation of a small pilot-scale continuous culture apparatus and gives data obtained during continuous growth of Aerobacter cloacae, for comparison with results predicted by theory.

\section{THEORY}

General principles of continuous flow systems

All continuous flow systems consist essentially of some form of reactor into which reactants flow at a steady rate and from which products emerge. The factors governing their operation are: (i) the way in which material passes through the reactor (which depends upon its design); (ii) the kinetics of the reaction taking place. As Danckwerts (1954) stated, (i) may be characterized by the distribution of residence-times of molecules or minute particles passing through the system. Most reactors lie between the extremes of the completelymixed tank and the ideal tubular type with 'piston flow' and no mixing. In ideal piston flow all particles have the same residence-time, equal to the mean residence-time, $\bar{t}$, while complete mixing produces a wide spread of residencetimes about the mean.

In a piston-flow reactor the extent of the reaction (whatever its kinetics) will be the same as for a batch reactor operated for a period equal to $\bar{t}$, while in a completely mixed reactor some of the material will have reacted for a considerably longer and some for a considerably shorter period than $\bar{t}$. Danckwerts (1954) pointed out that the piston flow reactor will be the more efficient for chemical reactions whose rates fall off as the reaction proceeds, but the completely-mixed reactor will be more efficient for reactions of the 'autocatalytic' type whose rate increases with time. Since bacterial growth is an autocatalytic process, the completely-mixed reactor will therefore be the most efficient type for continuous bacterial culture (a fact which has not always been appreciated), and only this type will be considered here.

Completely-mixed continuous cuiture apparatus. In the type of apparatus to be considered the reactor consists of some form of culture vessel in which the organism can be grown under suitable conditions. Sterile growth medium is fed into the culture vessel at a steady flow-rate $f$ and culture emerges from it at the same rate, a constant-level or similar device keeping the volume of culture in 
the vessel $(v)$ constant. The contents of the vessel are sufficiently well stirred to approximate to the ideal of complete mixing, so that the entering growth medium is instantaneously and uniformly dispersed throughout the vessel.

Residence-times in such a culture vessel will be determined not by the absolute values of the flow-rate and culture volume but by their ratio which we call the dilution rate, $D$, defined as $D=f / v$, i.e. the number of complete volume-changes $/ \mathrm{hr}$. The mean residence-time of a particle in the culture vessel is evidently equal to $1 / D$.

Assume for the moment that the bacteria in the culture vessel are not growing or dividing. With complete mixing, every organism in the vessel has an equal probability of leaving it within a given time. It can easily be shown to follow that the fraction of the total organisms in the vessel having a residence-time $\geqslant t$ is $\mathrm{e}^{-D t}$. The wash-out rate, i.e. the rate at which organisms initially present in the vessel would be washed out if growth ceased but flow continued is therefore:

$$
-\frac{\mathrm{d} x}{\mathrm{~d} t}=D x,
$$

where $x$ is the concentration of organisms in the vessel. The distribution of residence-times and the wash-out rate in a completely-mixed continuous culture vessel of this sort can thus be adequately described in fairly simple terms. Their application to continuous culture requires, in addition, a knowledge of the basic kinetics of the growth process.

\section{Kinetics of bacterial growth}

Theoretical discussions of bacterial growth usually start from the familiar 'exponential growth' equation

$$
\frac{1}{x} \frac{\mathrm{d} x}{\mathrm{~d} t}=\frac{\mathrm{d}\left(\log _{e} x\right)}{\mathrm{dt}}=\mu=\frac{\log _{e} 2}{t_{d}},
$$

where $x$ is the concentration of organisms (dry weight of organisms/unit volume) at time $t, \mu$ is the specific growth rate* and $t_{d}$ is the doubling time, $\dagger$ i.e. the time required for the concentration of organisms to double. In this equation, $\mu$ and $t_{d}$ are usually assumed to be constants; it is insufficiently appreciated, however, that this assumption is correct only when all substrates necessary for growth are present in excess.

Monod (1942) first showed that there is a simple relationship between the specific growth rate and the concentration of an essential growth substrate, $\mu$ being proportional to the substrate concentration when this is low but reaching a limiting saturation value at high substrate concentrations according to the equation

$$
\mu=\mu_{m}\left(\frac{s}{K_{s}+s}\right)
$$

* The actual rate of increase of concentration of organisms $(\mathrm{d} x / d t)$ is called the growth rate; the rate of increase/unit of organism concentration $\left(\frac{1}{x} \frac{\mathrm{d} x}{\mathrm{~d} t}\right)$ is called the specific growth rate.

$\dagger$ The doubling time is sometimes confused with the mean generation time, but strictly the two could only be identical if all organisms had identical individual generation times. 
where $s$ is the substrate concentration, $\mu_{m}$ is the growth rate constant (i.e. the maximum value of $\mu$ at saturation levels of substrate) and $K_{s}$ is a saturation constant numerically equal to the substrate concentration at which $\mu=\frac{1}{2} \mu_{m}$. It follows from equation (3) that exponential growth can occur at specific growth rates having any value between zero and $\mu_{m}$, provided the substrate concentration can be held constant at the appropriate value-a fact of major importance in continuous culture (Monod, 1950; Novick \& Szilard, 1950).

Monod (1942) also showed that there is a simple relationship between growth and utilization of substrate. This is shown in its simplest form in growth media containing a single organic substrate (e.g. glucose, ammonia and salts); under these conditions the growth rate is a constant fraction, $Y$, of the substrate utilization rate:

$$
\frac{\mathrm{d} x}{\mathrm{~d} t}=-Y \frac{\mathrm{d} s}{\mathrm{~d} t},
$$

where $Y$ is known as the yield constant. Thus over any finite period of growth

$$
\frac{\text { weight of bacteria formed }}{\text { weight of substrate used }}=\boldsymbol{Y} \text {. }
$$

If the values of the three growth constants $\mu_{m}, K_{s}$ and $Y$ are known, equations (2) to (4) provide a complete quantitative description of the 'growth cycle' of a batch culture (Monod, 1942). The same equations and constants are equally applicable to the theoretical treatment of continuous culture.

\section{Growth in continuous culture}

Experimental arrangement. Consider bacteria growing in a completely-mixed type of continuous culture vessel as described above, the inflowing medium containing a single organic substrate (e.g. glucose) at a concentration $s_{R}$. It is assumed that all other substrates are present in excess, and the culture vessel is so efficiently aerated that the oxygen supply is always adequate; the supply of organic substrate is therefore the sole growth-limiting factor. (Alternatively, conditions may be assumed to be completely anaerobic, when the same theoretical treatment will apply, though the actual values of $\mu_{m}$ and $Y$ will be different.) The variables within the control of the experimenter are the substrate concentration and flow rate of the incoming culture medium and a complete theory must describe how variation of these affects the growth rate and the concentrations of organisms and of substrate in the growth vessel.

Changes in concentration of organisms. In the culture vessel the organisms are growing at a rate described by equation (2) and simultaneously being washed away at a rate determined by equation (1). The net rate of increase of concentration of organisms is given by the simple balance equation (individual terms referring to rates in each case):

$$
\begin{aligned}
\text { increase } & =\text { growth }- \text { output }, \\
\frac{\mathrm{d} x}{\mathrm{~d} t} & =\mu x-D x .
\end{aligned}
$$


Hence if $\mu>D, \mathrm{~d} x / \mathrm{d} t$ is positive and the concentration of organisms will increase, while if $D>\mu, \mathrm{d} x / \mathrm{d} t$ is negative and the concentration of organisms will decrease, eventually to zero; i.e. the culture will be 'washed out' of the culture vessel. When $\mu=D, \mathrm{~d} x / \mathrm{d} t=0$ and $x$ is constant; i.e. we have a steady state in which the concentration of organisms does not change with time. Under such steady-state conditions, the specific growth rate, $\mu$, of the organisms in the culture vessel is exactly equal to the dilution rate $D$. This equation has been derived by several workers, but it has not always been realized that by itself the equation gives no information on what dilution rates make a steady state possible. To know this, we must also know how dilution rate affects the concentration of substrate in the culture vessel, since as already mentioned (equation 3) the value of $\mu$ depends on $s$.

Changes in substrate concentration. In the culture vessel, substrate is entering at a concentration $s_{R}$, being consumed by the organisms and flowing out at a concentration $s$. The net rate of change of substrate concentration is obtained by another balance equation (individual terms again referring to rates):

$$
\begin{aligned}
\text { increase } & =\text { input }- \text { output }- \text { consumption } \\
& =\text { input }- \text { output }-\frac{\text { growth }}{\text { yield constant }} \text { (from eqn. 4), } \\
\frac{\mathrm{d} s}{\mathrm{~d} t} & =D s_{R}-D s-\frac{\mu x}{\bar{Y}} .
\end{aligned}
$$

Fundamental equations of continuous culture. Equations (5) and (6) both contain $\mu$, which is itself a function of $s$ (equation 3). Substituting (3) in these equations we have:

from (5)

$$
\frac{\mathrm{d} x}{\mathrm{~d} t}=x\left\{\mu_{m}\left(\frac{s}{K_{s}+s}\right)-D\right\},
$$

and from (6)

$$
\frac{\mathrm{d} s}{\mathrm{~d} t}=D\left(s_{R}-s\right)-\frac{\mu_{m} x}{\boldsymbol{Y}}\left(\frac{s}{K_{s}+s}\right) .
$$

These are virtually identical with equations given by Monod (1950), though the above derivation is different from (and, we think, simpler than) Monod's. They define completely the behaviour of a continuous culture in which the fundamental growth relations are given by equations (2) to (4).

The steady state. It is apparent from consideration of equations (7) and (8) that if $s_{R}$ and $D$ are held constant and $D$ does not exceed a certain critical value (see equation 12), then unique values of $x$ and $s$ exist for which both $\mathrm{d} x / \mathrm{d} t$ and $\mathrm{d} s / \mathrm{d} t$ are zero; i.e. the system is in a steady state. Solving (7) and (8) for $\mathrm{d} x / \mathrm{d} t=\mathrm{d} s / \mathrm{d} t=0$, these steady-state values of $x$ and $s$, which will be designated $\tilde{x}$ and $\tilde{s}$ are given as

$$
\begin{aligned}
& \tilde{s}=K_{s}\left(\frac{D}{\mu_{m}-D}\right) \\
& \tilde{x}=Y\left(s_{R}-\tilde{s}\right)=Y\left\{s_{R}-K_{s}\left(\frac{D}{\mu_{m}-D}\right)\right\} .
\end{aligned}
$$


From these equations the steady-state concentrations of bacteria and substrate in the culture vessel can be predicted for any value of the dilution rate and concentration of inflowing substrate, provided the values of the growth constants $\mu_{m}, K_{s}$ and $\boldsymbol{Y}$ are known. They may be said to describe completely the behaviour of a continuous culture running under steady-state conditions. These equations also were first derived by Monod (1950).

While these equations describe accurately the situation existing once a steady state has become established, no proof was given by Monod that, starting from non-steady state conditions (such as exist at inoculation for example), a steady state must inevitably be reached. Rigorous proof of this has lately been provided by our colleague E. O. Powell who has shown (unpublished work) that, starting from any initial values of $x$ and $s$, the system inevitably adjusts itself to the steady state defined by equations (9) and (10), and that this is the only stable state of the system. For example, consider a system which has just been inoculated, when $x$ is very small, $s$ is nearly equal to $s_{R}$ and $\mu>D$. The concentration of organisms will increase, but owing to the resulting fall in substrate concentration the specific growth rate will decrease, until eventually $\mu$ becomes equal to $D$. At this point the combined rates of substrate consumption and loss just balance the rate of substrate addition and the system shows no further tendency to change. The system is stable in the sense that small accidental fluctuations from the steady-state values will set up opposing reactions which will restore the status quo. It is this automatic self-adjusting property of the system that makes continuous culture a readily feasible possibility.

As previously mentioned, in the steady-state the specific growth rate is equal to the dilution rate

$$
\mu=\frac{\log _{e} 2}{t_{d}}=\mu_{m}\left(\frac{\Im}{K_{s}+\tilde{s}}\right)=D .
$$

The doubling time $t_{d}$ is therefore equal to $0 \cdot 693 / D$; e.g. if one volume per hour is flowing through the culture vessel, the mass of organisms will be doubling every $42 \mathrm{~min}$.

As is evident from equations (9) and (10), the steady-state values of the concentrations of organisms and substrate depend solely on the values of $s_{R}$ and $\boldsymbol{D}$ (since $\mu_{m}, K_{s}$ and $\boldsymbol{Y}$ are constant for a given organism and growth medium). By varying $s_{R}$ and $D$ an infinite number of steady states can be obtained.

Effect of varying dilution rate. Fig. 1 shows how the mean generation time and the steady-state concentrations of bacteria and substrate in a continuous culture may be expected to vary when the dilution rate is varied, the inflowing substrate concentration being held constant; the curves are plotted from equations (9) and (10). The concentration of organisms has a maximum value when the dilution rate is zero, the substrate concentration then also being zero; i.e. the situation corresponds to the final stage of a batch culture. As the dilution rate increases the substrate concentration increases and the concentration of organisms falls, until at a critical value of $D$ the concentration of organisms becomes zero and the substrate concentration becomes equal to $s_{R}$; 
if plotted on appropriate scales the curves for bacterial and substrate concentrations are mirror-images.

The critical value of the dilution rate, above which complete 'wash-out' occurs, is obviously of great practical importance. This critical value, which is designated $D_{c}$, will be seen from equation (5) to be equal to the highest possible value of $\mu$, which is the value attained when $\tilde{s}$ has its highest possible value $s_{R}$, and is given by

$$
D_{c}=\mu_{m}\left(\frac{s_{R}}{K_{s}+s_{R}}\right) \text {. }
$$

When $s_{R} \gg K_{s}$, which is usually the case, then $D_{c} \sim \mu_{m}$.

At all dilution rates greater than $D_{c}, \mathrm{~d} x / \mathrm{d} t$ will be negative (from equation 5), bacteria will be washed out of the culture vessel faster than they can grow, and no steady state with $\tilde{x}>0$ is possible.

Effect of inflowing substrate concentration. In Fig. 2 the variation with the dilution rate of the steady-state concentrations of organisms and substrate is shown for a number of different values of the inflowing substrate concentration $s_{R}$; the curves are plotted from equation (9). It will be seen that at a given dilution rate below the critical the concentration of organisms is nearly proportional to $s_{R}$ (see equation 9 ), but the concentration of substrate is independent of $s_{R}$, i.e. the curve relating dilution rate to the concentration of substrate in the culture vessel is the same whatever the concentration of substrate in the inflowing culture medium (cf. Novick \& Szilard, 1950). In other words, when the dilution rate is fixed, the substrate concentration must come to a level (determined by equation 9 ) that makes $\mu$ equal to $D$, and this level is independent of $s_{R}$.

The curve relating concentration of organisms to dilution rate is seen (Fig. 2) to be displaced vertically as $s_{R}$ increases, the drop in $\tilde{x}$ at high dilution rates being steeper for higher values of $s_{R}$. Consideration of equation (3) shows that the important factor here is not the absolute value of $s_{R}$ but the value of the ratio $s_{R} / K_{s}$. The higher this ratio, the greater the fraction of total substrate that can be consumed without an appreciable decrease in the specific growth rate. Hence as $s_{R} / K_{s}$ is increased the concentration of organisms is maintained at nearly the maximum value up to higher values of $D$ and the critical dilution rate $D_{c}$ approaches more closely to $\mu_{m}$ (cf. equation 12). The value of $K_{s}$ is very low $\left(10^{-4} \mathrm{M}\right.$ or less) for most substrates, so that $s_{R} / K_{s}$ ratios will usually be high in practice.

Performance criteria: output and yield. When a continuous culture system is viewed as a production process, its performance may be judged by two criteria: (i) the quantity of bacteria produced in unit time, which will be called the output rate; (ii) the quantity of bacteria produced from unit weight of substrate, which will be called the effective yield.

The total output from a continuous culture unit in the steady state is obviously equal to the product of flow-rate and concentration of organisms, or $f \tilde{x}$; the output/unit volume of culture is therefore $D \tilde{x}$, and from equation (10):

$$
\text { Output }=D \tilde{x}=D Y\left\{s_{R}-K_{s}\left(\frac{D}{\mu_{m}-D}\right)\right\} \text {. }
$$


Now as $D$ is increased from 0 to $D_{c}, \tilde{x}$ decreases from $Y s_{R}$ to 0 , and it can be shown that there is a value of $D$ for which the product $D \tilde{x}$ is a maximum; in other words, for any system there is a particular dilution rate, $D_{M}$, which gives the maximum output of organisms in unit time. This maximum output value of $D$ is obtained by differentiating equation (13) with respect to $D$ and equating to zero, and is

$$
D_{M}=\mu_{m}\left\{1-\sqrt{\frac{K_{s}}{K_{s}+s_{R}}}\right\}
$$

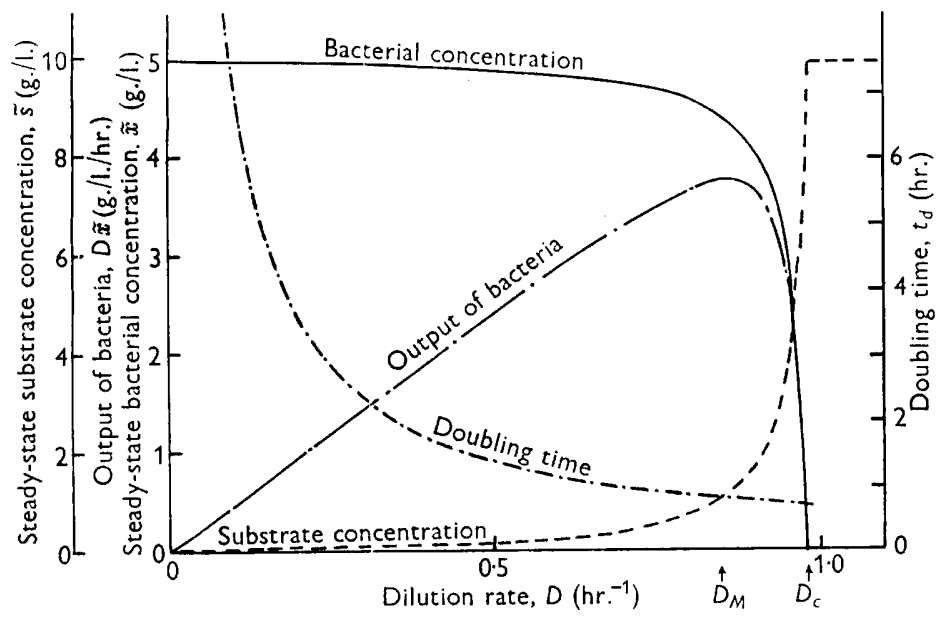

Fig. 1. Steady-state relationships in a continuous culture (theoretical). The steady-state values of substrate concentration, bacterial concentration and output at different dilution rates are calculated from equations (9) and (10), for an organism with the following growth constants: $\mu_{m}=1.0 \mathrm{hr} .^{-1}, Y=0.5$ and $K_{s}=0.2 \mathrm{~g} . / 1$; and a substrate concentration in the inflowing medium of $s_{R}=10 \mathrm{~g}$. $/ \mathrm{l}$.

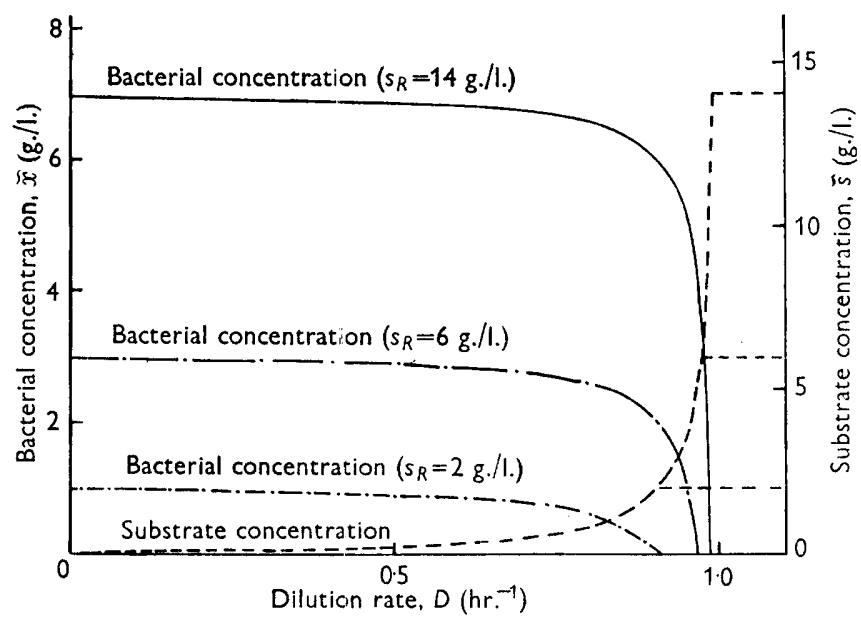

Fig. 2. Effect of varying the concentration of substrate in the inflowing medium $\left(s_{R}\right)$ on the steady-state relationships in a continuous culture (theoretical). The curves are calculated from equations (9) and (10) for an organism with $\mu_{m}=1.0 \mathrm{hr} .^{-1}, Y=0.5$, and $K_{s}=0 \cdot 1 \mathrm{~g} . / \mathrm{l}$, for media of three different substrate concentrations. 
The steady-state concentration of organisms at this dilution rate is obtained by substituting the above value of $D_{M}$ in equation (10) and is

$$
\tilde{x}_{M}=Y\left\{\left(s_{R}+K_{s}\right)-\sqrt{ }\left(K_{s}\left(s_{R}+K_{s}\right)\right)\right\} .
$$

The maximum output rate, $D_{M} \tilde{x}_{M}$, is the product of equations (14) and (15) and is given below (equation 23).

Output of organisms is plotted against dilution rate in Fig. 1. The output is nearly proportional to $D$ at low $D$ values (up to about $0.5 D_{c}$ ); as $D$ increases the output curve flattens, reaches a maximum and falls rapidly to zero at the critical dilution rate.

The yield constant $Y$, defined as in equation (4), is related to the steadystate concentrations of organisms and substrate in a continuous culture by the expression

$$
\boldsymbol{Y}=\frac{\text { output of bacteria }}{\text { substrate utilized }}=\frac{\tilde{x}}{s_{R}-\tilde{s}} .
$$

The effective yield, $Y_{E}$, is defined as the ratio of bacteria formed to substrate supplied in the inflowing culture medium and is given by

$$
Y_{E}=\frac{\text { output of bacteria }}{\text { input of substrate }}=\frac{\tilde{x}}{s_{R}}=\frac{Y\left(s_{R}-\tilde{s}\right)}{s_{R}} .
$$

At all flow-rates $>\mathbf{0}$, the effective yield is less than the yield constant owing to the substrate wasted in the outflow, $s$, which increases with the dilution rate (Fig. 1).

The efficiency of utilization of the substrate supplied in the inflowing growth medium is given by equations (9) and (17) as

$$
\frac{Y_{E}}{Y}=\frac{s_{R}-\tilde{s}}{s_{R}}=\frac{s_{R}-K_{s}\left(\frac{D}{\mu_{m}-D}\right)}{s_{R}} .
$$

It follows that for maximum utilization of substrate the dilution rate should be as low as possible; this is, strictly speaking, incompatible with a maximum output of bacteria, which requires the dilution rate to be high $\left(D_{M}\right.$ being close to $D_{c}$ ). However, the shape of the curve relating $\tilde{s}$ to $D$ (Fig. 1 ) is such that loss of substrate in the outflow is in practice negligible up to quite high dilution rates; in the example plotted utilization of substrate is $>95 \%$ complete at all dilution rates up to $0 \cdot 7 D_{c}$, and is still $90 \%$ complete at the maximum output rate $D_{M}$.

The efficiency of utilization of substrate at the maximum output rate $D_{M}$ is given by equations (14) and (18) as

$$
\frac{s_{R}-\tilde{s}_{M}}{s_{R}}=\frac{\left(s_{R} / K_{s}+1\right)-\sqrt{ }\left(s_{R} / K_{s}-1\right)}{s_{R} / K_{s}} .
$$

The efficiency of utilization will be seen to depend solely on the ratio $s_{R} / K_{s}$ and approaches $100 \%$ if this ratio is made sufficiently high; in other words, high substrate concentrations are advantageous for efficient utilization of substrate.

To summarize, the conditions for maximum production efficiency, combining a high output with efficient utilization of substrate, will be obtained 
with a flow-rate at or a little below the maximum output rate $D_{M}$ and the highest practicable substrate concentration. It must be emphasized, however, that these are the optimum conditions when the object is to produce microorganisms. When the desired product is a fermentation product whose formation is proportional to the amount of substrate breakdown (e.g. ethanol, lactic acid), optimum conditions should be much the same, but they may be widely different for the production of complex metabolic products such as antibiotics or exotoxins.

Comparison of continuous and batch culture. The relative outputs of continuous and batch cultures are of interest from the production standpoint. The output of a batch culture of course varies throughout the growth cycle, but a mean output can be calculated as follows.

Consider a batch of medium of initial substrate concentration $s_{\mathbf{0}}$, inoculated initially with organisms to a concentration $x_{0}$; the maximum growth attained when all substrate has been utilized is $x_{m}$. Then the total time of one production cycle is

$$
t=\frac{1}{\mu_{m}} \log _{e} \frac{x_{m}}{x_{0}}+t_{L}
$$

where the first term is the time which would be occupied if the organisms grew exponentially at maximum rate from start to finish, and the second term $t_{L}$ is an overall 'delay time' which includes the initial lag and final retardation phases of growth and the 'turnaround time' necessary to take down, sterilize and re-assemble the plant preparatory to a second cycle.

The total amount of organisms produced (from equation 3 ) is

$$
x_{m}-x_{0}=Y s_{0}\left(\frac{x_{m}-x_{0}}{x_{m}}\right) .
$$

The mean output is therefore

$$
\frac{\text { total organisms produced }}{\text { total cycle time }}=\frac{\mu_{m} Y s_{0}\left(\frac{x_{m}-x_{0}}{x_{m}}\right)}{\log _{e} \frac{x_{m}}{x_{0}}+\mu_{m} t_{L}}
$$

The maximum output of a continuous culture is from equations (14) and (15):

$$
D_{M} \tilde{x}_{M}=\mu_{m} Y s_{R}\left\{\sqrt{\frac{K_{s}+s_{R}}{s_{R}}}-\sqrt{\frac{K_{s}}{s_{R}}}\right\}^{2}
$$

where the term in brackets has a value close to $1 \cdot 0$ for high $s_{R} / K_{s}$ ratios such as obtain in practice and may therefore be neglected. Hence, when the same growth medium is used in both cases, so that $s_{0}=s_{R}$, we have from equations (22) and (23)

$$
\frac{\text { continuous output }}{\text { batch output }}=\frac{\log _{e} \frac{x_{m}}{x_{0}}+\mu_{m} t_{L}}{\left(x_{m}-x_{0}\right) / x_{m}}
$$

The value of this ratio is affected mainly by the growth rate of the organism and the total delay time, inoculum size having a lesser effect. Assuming 
a ' $5 \%$ inoculum' $\left(x_{0} / x_{m}=0.05\right)$, and a delay time $t_{L}$ of $6 \mathrm{hr}$., calculations from (24) give the following results:

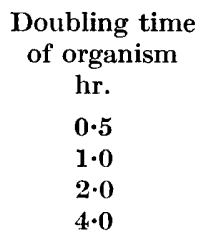

\begin{tabular}{c} 
Ratio: \\
continuous output \\
\hline batch output \\
$11 \cdot 9$ \\
$7 \cdot 6$ \\
$5 \cdot 3$ \\
$4 \cdot 3$
\end{tabular}

The delay time of $6 \mathrm{hr}$. assumed in these calculations is fairly optimistic; both plant turn-around time and growth lag would exceed this in many instances, which would further increase the above ratios. In the majority of cases, therefore, a continuous process would be expected to show at least a five- to tenfold advantage over the corresponding batch process.

\section{APPARATUS AND METHODS}

Continuous culture apparatus. The apparatus used is of conventional small pilot plant type and is fabricated wholly in stainless steel; the layout is shown in Fig. 3. It consists of two sterilizing tanks $S_{1}, S_{2}$ (each of 300 l. working volume) connected in parallel and used alternately to feed growth medium into the culture vessel, $C V$. This has a working capacity of $20 \mathrm{l}$. and is of conventional design; mixing and aeration are effected by a vane-disk impeller mounted on a central shaft passing through a stuffing-box in the lid, with wall baffles and injection of sterile air immediately under the impeller, similar to the arrangement of Chain et al. (1954). The oxygen transfer rate, measured by the method of Cooper, Fernstrom \& Miller (1944), is $135 \mathrm{mmole}_{2} / \mathrm{l}$./hr. with an air flow of $1.0 \mathrm{vol} . / \mathrm{vol}$. culture/min. The level of culture in the vessel is maintained constant by a side overflow tube through which the culture flows by gravity into two calibrated collecting tanks $M_{1}, M_{2}$, which are connected in parallel, and thence to a large holding tank $H$. Effluent air leaves the culture vessel by a separate outlet; both entering and exit air is sterilized by heat. Air and medium flow-rates are indicated by Rotameters $R_{1}, R_{2}$, and are controlled by manual adjustment of cocks; a second check on medium flow-rate is provided by the calibrated tanks $M_{1}, M_{2}$. Sampling points $S P_{1-2}, S P_{3}$, are provided on the sterilizers and culture vessel respectively. On the culture vessel there is a point $\boldsymbol{A P} \boldsymbol{P}_{\mathbf{1}}$ for the addition of inoculum and anti-foam ('Antifoam A', Midland Silicones Ltd.). Temperature is controlled by circulating thermostatically controlled water through an internal coil (not shown in diagram) in the culture vessel and was $37^{\circ}$ for all experiments described in this paper.

Operation. The whole equipment is sterilized empty with internal steam at $20 \mathrm{lb}$./sq.in., followed by filling and sterilization of the medium reservoirs. The culture vessel is then charged with $20 \mathrm{l}$. of sterile medium and inoculated with 1 1. of a shake-flask culture of the selected organism grown on the same medium. Growth is allowed to proceed batchwise until the concentration of organisms 
reaches $90 \%$ of the expected peak value; medium flow through the culture vessel is then started at a selected value and the culture from then on run continuously. Samples of sterile medium are taken from each sterilizing tank for analysis and samples of the culture are usually taken hourly; samples of the exit air are also taken for gas analysis.

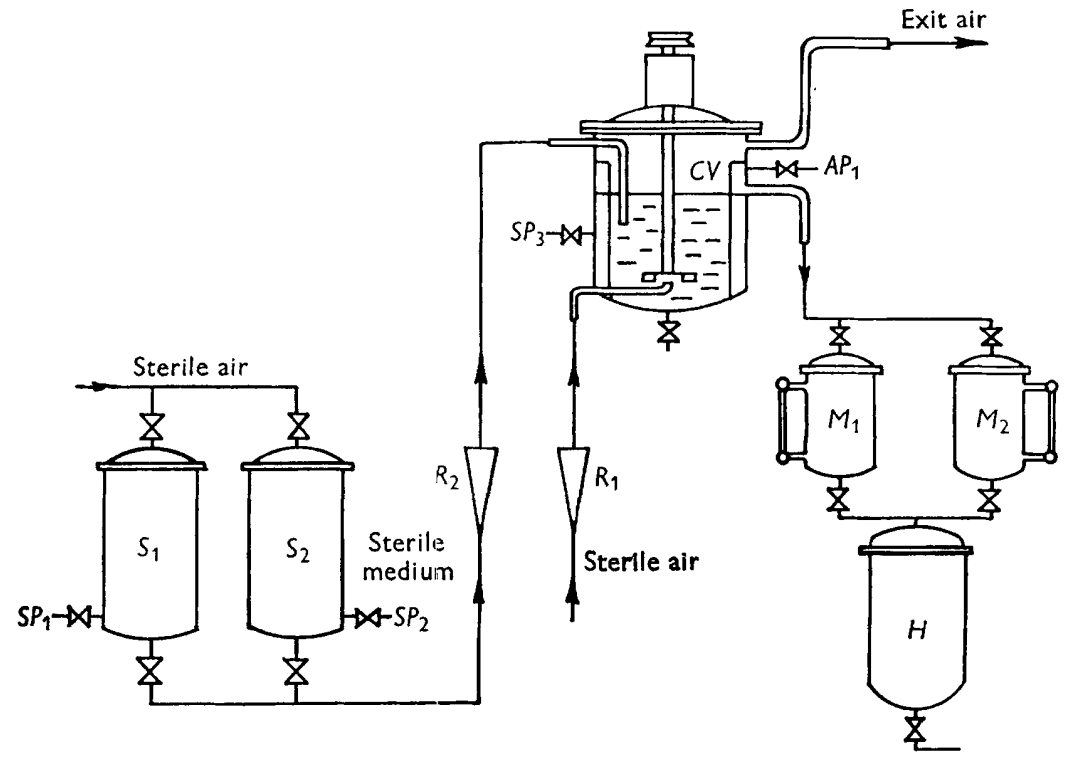

Fig. 3. Pilot plant for continuous culture of bacteria (schematic). $S_{1}, S_{2}$, sterilizing tanks (300 1. working volume); $C V$ culture vessel (20 1. working volume); $M_{1}, M_{2}$, measuring tanks (30 l.); $H$, holding tank; $R_{1}, R_{2}$, rotameters; $S P_{1}, S P_{2}, S P_{3}$, sampling points; $A P_{1}$, anti-foam addition point.

Observations and measurements. Each batch of medium was examined or analysed for sterility, $\mathrm{pH}$ value, ammonia- $\mathrm{N}$ content and concentration of organic substrate, which in the present instance was glycerol, determined according to Neish (1952). Samples from the culture vessel were tested for $\mathrm{pH}$ value, concentration of glycerol, total count (by Helber counting chamber), viable count (by the Miles \& Misra (1938) technique on plates of Hartley's digest agar) and purity of the microbial species being grown.

Organisms and culture mediuri. Aerobacter cloacae (Cloaca cloacae strain NCTC 8197) was used throughout and grown in medium of the following composition: $0.09 \mathrm{M}-\left(\mathrm{NH}_{4}\right)_{2} \mathrm{HPO}_{4}, 0.01 \mathrm{M}-\mathrm{NaH}_{2} \mathrm{PO}_{4}, 0.01 \mathrm{M}-\mathrm{K}_{2} \mathrm{SO}_{4}, 0.001 \mathrm{M}-\mathrm{MgSO}_{4}$, $0.0001 \mathrm{M}-\mathrm{CaCl}_{2}, 0.00002 \mathrm{M}-\mathrm{FeSO}_{4}$, and $0.0272 \mathrm{M}(0.25 \%$, w/v) glycerol; the $\mathrm{pH}$ value after sterilizing was $7 \cdot 3-7 \cdot 4$. In this medium glycerol (selected in preference to glucose for its stability on sterilization) was the sole carbon source and was also the growth-limiting component, all other components being present in excess. A rather low concentration was used to ensure fairly low bacterial concentrations so that aeration was always adequate. 


\section{RESULTS}

\section{Growth in batch culture}

As a preliminary to continuous culture studies, data were obtained on the growth of the organism in batch culture, using the same apparatus and growth medium. Typical experiments are shown in Fig. 4. After a variable lag period, which depends on the age and size of the inoculum, growth proceeds exponentially until almost all the substrate has been exhausted (a sign that aeration is not rate-limiting). Under these conditions the organisms are growing for most of the time in substrate concentrations which are high compared with $K_{s}$, and equation (3) becomes

$$
\frac{1}{x} \frac{\mathrm{d} x}{\mathrm{~d} t}=\frac{\mathrm{d}\left(\log _{e} x\right)}{\mathrm{d} t} \sim \mu_{m} .
$$

The value of $\mu_{m}$ is given by the slope of the straight line obtained by plotting $\log _{e} x$ against $t$, the best straight line being fitted by the 'method of least squares'. The mean value obtained from a number of experiments was $\mu_{m}=0.85 \mathrm{hr}^{-1}$ (doubling time $t_{d}=49 \mathrm{~min}$.).

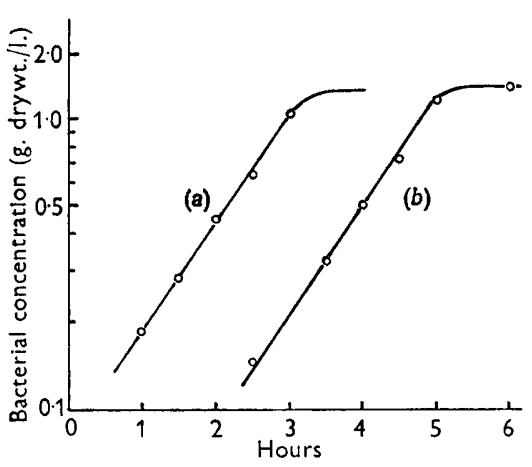

Fig. 4

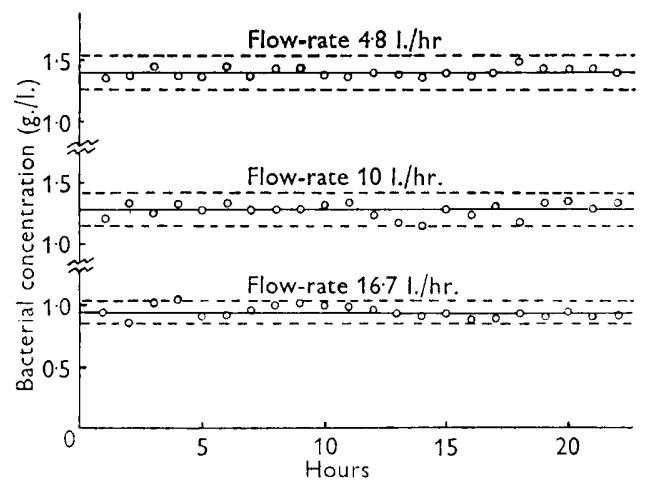

Fig. 5

Fig. 4. Growth curves of Aerobacter cloacae in batch culture. Details of growth conditions and culture medium are given in the text. Curve $(a)$ was obtained from a seed culture at the start of a continuous culture run; curve $(b)$ was obtained after the culture had been operated continuously for 6 weeks (the points on curve $(b)$ have been displaced $2 \mathrm{hr}$. to the right to avoid overlapping with curve $(a)$ ).

Fig. 5. Steady states in continuous culture. Aerobacter cloacae was grown in continuous culture as described in the text; the data plotted are hourly measurements of bacterial concentration at three different flow-rates. The continuous lines are the lines of best fit, calculated statistically; the dotted lines are the $95 \%$ fiducial limits.

In the medium used, the total growth $\left(x_{m}-x_{0}\right)$ obtained in a batch culture was strictly proportional to the initial concentration of glycerol in the medium (cf. Monod, 1942). The mean of several determinations of the yield constant was $Y=0.53 \mathrm{~g}$. dry weight of organism/g. glycerol used; this corresponds to a $61 \%$ conversion of the glycerol carbon to bacterial carbon. (The carbon content of the bacteria was found to be $45 \%$ of the dry weight.) 
Growth in continuous culture

Steady-state operation. In accordance with theory, steady-state operation was found to be possible over a wide range of flow-rates, the range actually tested being from 4 to $22 \mathrm{l}$./hr., corresponding to dilution rates of $0 \cdot 2-1 \cdot 1 \mathrm{hr} .^{-1}$. The critical flow-rate for complete wash-out (obtained by extrapolation of the curves of Fig. 6 ) was about $24 \mathrm{l}$./hr., corresponding to a critical dilution rate of about $1 \cdot 2 \mathrm{hr} .^{-1}$.

Over the stable range of flow-rates the culture was self-adjusting; i.e. on setting the flow-rate to a given value, the concentrations of organisms and substrate would move towards and settle down at steady levels which were maintained indefinitely so long as the flow-rate remained unaltered; on changing the flow-rate, new steady-state levels were automatically attained. After a change of flow-rate, some hours might elapse before the culture had stabilized at the new steady state, particularly when the change in $D$ was large. The culture was therefore always run for at least $24 \mathrm{hr}$. after a flow-rate change, before measurements at the new steady state were begun.

Examples of steady states at three different flow-rates are given in Fig. 5; measurements of bacterial concentration were taken hourly for $24 \mathrm{hr}$., after a preliminary $24 \mathrm{hr}$. stabilization period. Statistical analysis of the results showed that the plots of bacterial concentration against time did not differ significantly from straight lines of zero slope; the apparent small fluctuations in bacterial concentration were purely random and within the errors of measurement (95\% fiducial limits for each curve are shown by the dotted lines).

Maintenance of purity of culture. Under this heading we include both contamination of the culture with foreign micro-organisms and mutation of the parent organism to an extent sufficient to alter the characteristics of the culture. To detect such occurrences, samples taken directly from the culture vessel were repeatedly examined $(a)$ microscopically, $(b)$ by plating out and examination of colonies, $(c)$ by regular subculturing and examination by biochemical tests of typical colonies (and any atypical ones, when observed).

Such tests readily detect gross contamination, but statistical considerations show that they are not very efficient in revealing small degrees of contamination. Suppose that in the whole culture there is an average of $z$ contaminants to every $n$ total organisms. If $z$ is small its distribution in samples of $n$ will be Poissonian; i.e. the probability $(\boldsymbol{P})$ of finding $r$ contaminants in a single sample of $n$ cells is $\mathrm{e}^{-z} z^{r} / r$ !, and the probability of finding no contaminant at all is $\mathrm{e}^{-z}$. Hence in $5 \%$ of cases no contaminants will be observed when the expected number is three $\left(\boldsymbol{P}=\mathrm{e}^{-3}=0.05\right)$. For example, even if no contaminants are found in a sample of 1000 colonies examined, there is still a $5 \%$ chance that the degree of contamination is really as high as $0.3 \%$. The same considerations apply in any form of sterility testing and are discussed in more detail by Elsworth, Telling \& Ford (1955).

It is therefore practically impossible to show that a culture is completely free from contaminants by a single test. Repeated tests over long periods are more significant, however, since the permanent existence of a low equilibrium 
level of contaminants is improbable; foreign contaminants (or mutants) are most likely either to displace the parent culture altogether, or to disappear.

With the above reservations it can be said that over the past two years no difficulty has been found in operating the continuous culture apparatus for long periods without any detectable contamination. The longest individual run lasted 108 days and was still free from contamination when terminated voluntarily; 2-3 months is the average period for most runs. The construction of a leak-free apparatus did not prove too difficult, and contaminations experienced in the earlier stages of the work could usually be ascribed to defective initial sterilization or faulty aseptic technique during sample-taking or addition of anti-foam. If due attention be paid to these points, we see no reason why continuous cultures should not be maintained free from contamination more or less indefinitely.

So far as mutations are concerned, we have never during the longest periods of continuous operation been able to detect any organisms differing from the parent strain in microscopic or colonial morphology, biochemical reactions or growth characteristics. In view of the possibility of faster-growing though morphologically identical mutants displacing the parent strain more or less completely, we have on numerous occasions re-determined the value of the growth-rate constant $\mu_{m}$ at intervals during the course of a continuous culture. This can easily be done by stopping the medium flow, draining off $95 \%$ or more of the culture ( the remainder serving as inoculum), re-filling the culture vessel with fresh medium and growing up as a batch culture, the growth rate and yield constant being determined as in Fig. 4. No significant change in $\mu_{m}$ or $\boldsymbol{Y}$ was ever observed, even after long periods of continuous culture (cf. curves $(a)$ and (b) of Fig. 4).

Quantitative tests of continuous culture theory. Table 1 summarizes quantitative data on steady-state bacterial and substrate concentrations at twenty-one different flow-rates. These were all obtained during a single run, over a period of 65 days continuous operation. In Fig. $6(a)-(d)$ the steady-state bacterial concentration and output, substrate concentration and yield constant are plotted against flow-rate, for comparison with the results to be expected according to Monod's theory.

The theoretical curves shown were plotted from equations (9) and (10), using the values $\mu_{m}=0.85 \mathrm{hr}^{-1}$ and $Y=0.53$ obtained in the batch culture experiments. Determination of $\boldsymbol{K}_{s}$ was more difficult. Accurate values are not readily obtained from batch culture experiments, since at the low substrate concentrations necessary, $s$ is continually decreasing during the experiment. Theoretically, $K_{s}$ is most easily determined from continuous culture experiments, for a single measurement of the steady-state substrate concentration at any dilution rate allows $K_{s}$ to be calculated if $\mu_{m}$ is known, as is shown by re-arranging equation (9) in the form

$$
K_{s}=\tilde{s}\left(\frac{\mu_{m}-D}{D}\right) .
$$

In particular, when $D=\mu_{m} / 2$, we have $\tilde{s}=K_{s}$, and this is probably the most accurate method for determining $K_{s}$, provided the analytical method for 
determining $\tilde{s}$ is sufficiently sensitive. In the present case, unfortunately, the value of $\boldsymbol{K}_{s}$ was so small that over most of the lower range of dilution rates the substrate concentration was too low to measure with the available methods for glycerol estimation (Table 1 ). $\boldsymbol{K}_{\boldsymbol{s}}$ was therefore determined from equation (14), using experimentally determined values of $\mu_{m}$ and the maximum output

\section{Table 1. Quantitative data on the continuous culture of Aerobacter cloacae}

The apparatus and general plan of the experiment are described in the text. Each row of figures in the table refers to a different steady state. Glycerol concentration in the inflowing culture medium $\left(s_{R}\right)$ was $2 \cdot 5 \mathrm{~g} . / 1$.

\begin{tabular}{|c|c|c|c|c|c|}
\hline $\begin{array}{c}\text { Flow } \\
\text { rate } \\
(1 . / h r .)\end{array}$ & $\begin{array}{c}\text { Duration* } \\
\text { of test }\end{array}$ & $\begin{array}{c}\text { Bacterial } \\
\text { concentration } \\
\text { (g. dry wt./l.) }\end{array}$ & $\begin{array}{c}\text { Output of } \\
\text { bacteria } \\
\text { (g./hr.) }\end{array}$ & $\begin{array}{c}\text { Glycerol } \\
\text { concentration } \\
\text { in culture } \\
(\mathrm{g} . / \mathrm{l} .)\end{array}$ & $\begin{array}{c}\text { Yield } \\
\text { constant } \\
\text { (g. bacter } \\
\text { g. glycer }\end{array}$ \\
\hline$f$ & (hr.) & $\tilde{\boldsymbol{x}}$ & $f \tilde{x}$ & $\tilde{\boldsymbol{s}}$ & $\frac{\tilde{x}}{s_{R}-\tilde{s}}$ \\
\hline 0 & $\begin{array}{l}\text { (Batch } \\
\text { culture) }\end{array}$ & $1 \cdot 32 \dagger$ & - & 0 & $0.53 \dagger$ \\
\hline $4 \cdot 6$ & 22 & $1+33$ & $6 \cdot 12$ & $<0.03 \ddagger$ & 0.55 \\
\hline 4.8 & 26 & $1 \cdot 39$ & $6 \cdot 66$ & $<0.03$ & 0.57 \\
\hline $5 \cdot 0$ & 25 & $1 \cdot 30$ & $6 \cdot 48$ & $<0.03$ & 0.53 \\
\hline $7 \cdot 2$ & 25 & $1 \cdot 26$ & $9 \cdot 05$ & $<0.03$ & 0.52 \\
\hline $8 \cdot 4$ & 14 & $1 \cdot 25$ & $10 \cdot 47$ & - & - \\
\hline $10 \cdot 0$ & 25 & 1.29 & $12 \cdot 88$ & $<0.03$ & 0.53 \\
\hline 11.5 & 22 & $1 \cdot 81$ & $15 \cdot 04$ & $<0.03$ & 0.54 \\
\hline $11 \cdot 9$ & 24 & $1 \cdot 27$ & $15 \cdot 08$ & - & - \\
\hline $13 \cdot 8$ & 23 & 1.23 & $16 \cdot 92$ & - & - \\
\hline $13 \cdot 9$ & 34 & $1 \cdot 2 \cdot 2$ & $17 \cdot 0$ & $<0.03$ & 0.50 \\
\hline $14: 3$ & 16 & $1 \cdot 04$ & $14 \cdot 9$ & $<0.03$ & $0 \cdot 43$ \\
\hline $14 \cdot 5$ & 17 & $1 \cdot 07$ & $15 \cdot 5$ & - & 一 \\
\hline $15 \cdot 7$ & 25 & 0.96 & $15 \cdot 1$ & $<0.03$ & $0 \cdot 40$ \\
\hline $15 \cdot 8$ & 23 & $1 \cdot 26$ & $19 \cdot 0$ & $<0.03$ & $0 \cdot 52$ \\
\hline $15 \cdot 9$ & 20 & 0.91 & $14 \cdot 3$ & $<0.03$ & $0 \cdot 36$ \\
\hline $16 \cdot 6$ & 22 & $1 \cdot 00$ & $16 \cdot 5$ & - & - \\
\hline $16 \cdot 7$ & 24 & 0.913 & $16 \cdot 3$ & 0.26 & $0 \cdot 44$ \\
\hline $17 \cdot 3$ & 12 & 0.95 & $16 \cdot 4$ & $0 \cdot 28$ & $0 \cdot 43$ \\
\hline $18 \cdot 3$ & 8 & 0.72 & $13 \cdot 1$ & $0 \cdot 88$ & $0 \cdot 45$ \\
\hline $19 \cdot 5$ & 6 & 0.35 & $6 \cdot 84$ & $1 \cdot 66$ & $0 \cdot 45$ \\
\hline $20 \cdot 0$ & 9 & $0 \cdot 48$ & $9 \cdot 68$ & $1 \cdot 37$ & 0.42 \\
\hline $22 \cdot 4$ & 8 & $0 \cdot 24$ & $5 \cdot 31$ & $\mathbf{1 . 9 3}$ & $0 \cdot 34$ \\
\hline
\end{tabular}

* The culture was allowed to stabilize for 18-24 hr. at each flow-rate before observations commenced; it was then sampled hourly during the test period. The figures for bacterial and glycerol concentrations are the means of the hourly samples.

$\dagger$ Means of a number of batch culture experiments.

$\ddagger$ This figure represents the lowest concentration of glycerol which can be measured with accuracy by the method used.

dilution rate $D_{M}$ and solving for $K_{s}$. The value obtained, which may be subject to considerable error, was $K_{s}=1 \cdot 35 \times 10^{-4} \mathrm{M}$, or $12 \cdot 3 \mu \mathrm{g}$. glycerol $/ \mathrm{ml}$. This is comparable with the values of $K_{s}$ found by Monod (1942) for other carbohydrate substrates (namely 4, 2 and $20 \mu \mathrm{g} . / \mathrm{ml}$. for glucose, mannitol and lactose respectively), but considerably higher than the value of $10^{-3} \mu \mathrm{g} . / \mathrm{ml}$. found by Novick \& Szilard (1950) with tryptophan as substrate. The above values of $\mu_{m}, K_{s}$ and $Y$ were used in calculating the 'theoretical' curves shown in Fig. 6. 
Qualitatively, the results plotted in Fig. 6 may be seen to be in general agreement with the theory. The steady-state concentration of organisms falls only very slowly as the dilution rate is increased from zero to about $0 \cdot 8 \mathrm{hr} .^{-1}$ (Fig. 6a); as the flow-rate is increased still further the bacterial concentration drops fairly steeply, the curve extrapolating to complete wash-out at $D=D_{c}=1.2 \mathrm{hr}^{-1}$ (approx.). The output of bacteria (Fig. $6 \mathrm{~b}$ ) is nearly proportional to the flow-rate up to a maximum at $D_{M}=0.79 \mathrm{hr}^{-1}$, thereafter falling fairly sharply to zero. The curve for steady-state glycerol concentration (Fig. 6c) should be a mirror-image of that for bacterial concentration (cf. Figs. 1
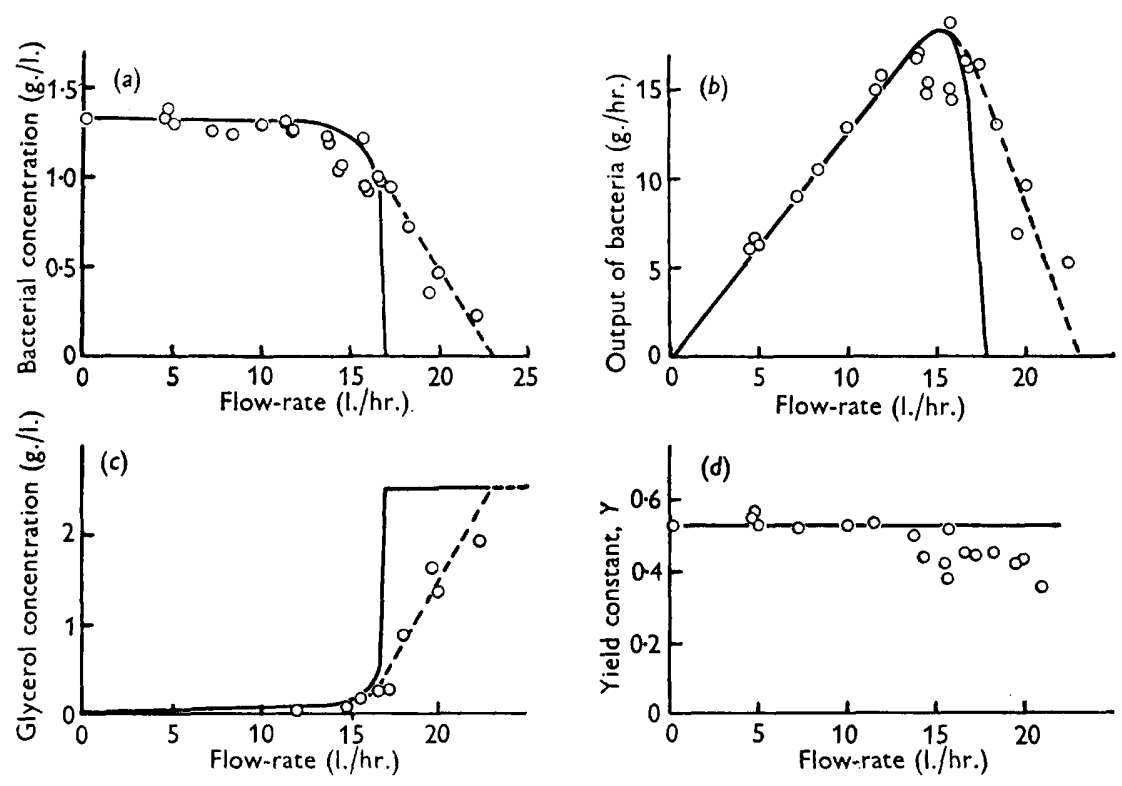

Fig. 6. Quantitative relationships in continuous culture of Aerobacter cloacae; comparison of experiment with theory. The data of Table 1 are plotted for comparison with the theoretical relationships calculated from equations (9) and (10). The continuous lines are the theoretical curves; the dotted lines are drawn to fit the experimental points.

and 2), and this is seen to be approximately the case; at all flow-rates up to about $16 \mathrm{l} / \mathrm{hr}$., however, utilization of substrate was so nearly complete that the residual glycerol was too low for accurate measurement.

Quantitatively, agreement with theory is good at all the lower flow-rates (below $D=0.8 \mathrm{hr}^{-1}$ ), discrepancies being less than the experimental errors of bacterial dry weight and glycerol determinations. At the higher flow-rates, however, there are definite discrepancies; the curves for bacterial concentration and output and for glycerol concentration, though of the right general shape, show definite deviations from the predicted curves, bacterial concentrations being higher and substrate concentrations being lower than the values expected. In particular, the observed value of the critical dilution rate is definitely higher than that calculated from equation (12), and genuine steadystate conditions appear to have been achieved at dilution rates with $D>\mu_{m}$, 
when theory predicts that complete 'wash-out' should occur. Another discrepancy is that the yield constant $Y$, which at low dilution rates has the same value as was found in batch culture experiments, shows a definite tendency to decrease at higher dilution rates (Fig. 6d). Possible reasons for these divergences are discussed below.

\section{DISCUSSION}

One object of the present work was to compare the results obtained during the operation of a continuous culture apparatus with those predicted by the theory given earlier. It is also relevant to compare them with the results predicted by the theories of other workers.

A common feature of all theories of continuous culture is the elementary one that net growth must be the resultant of exponential growth minus exponential 'wash-out', or re-writing equation (5):

$$
\frac{\mathrm{d}\left(\log _{e} x\right)}{\mathrm{d} t}=\mu-D \text {. }
$$

Hence when a steady state $\left(d\left(\log _{e} x\right) / \mathrm{d} t=0\right)$ exists, then $\mu=D$, i.e. the specific growth rate must be equal to the dilution rate. An error made by a number of authors in mathematical discussions of continuous culture is to confuse, in the above equation, the specific growth rate $\mu$ (which varies with the substrate concentration) and the growth rate constant $\mu_{m}$. Obviously, if one puts in equation (27) $\mu=\mu_{m}=$ constant, it follows that a steady state is possible only at one particular flow-rate, when $D=\mu_{m}$. This is the assumption made by Golle (1953) who states that 'there is only one rate of medium flow. . . at which steady-state conditions will be maintained'. Finn \& Wilson (1954) also stress this point, and the same idea appears to be implicit in the writings of Adams \& Hungate (1950) and Northrop (1954). If this were so, a continuous culture would be an inherently unstable system and very difficult to operate; moreover, restriction to a single flow-rate would greatly limit its usefulness. The experimental data presented in this paper show conclusively that a continuous culture is an inherently stable system adjusting itself automatically to changes in dilution rate. Any number of steady states can be obtained at different dilution rates anywhere between zero and the critical value, as the theories of Monod (1950) and Novick \& S zilard (1950) predict.

The essential features of the Monod-Novick \& Szilard theories and the theoretical treatment given in this paper* is that they take into account the observed facts that bacteria can grow only at the expense of the substrate utilized, and that their specific growth rate is a function of the substrate concentration. It then becomes apparent that a continuous culture apparatus is a device for controlling growth through control of the substrate concentration; each dilution rate fixes the substrate concentration at that value which makes $\mu$ equal to $D$.

* We wish to emphasize that the theoretical treatment given in this paper is merely an expansion and development of those of Monod and of Novick \& Szilard (particularly the former), and is based on the fundamental principles which they originated. 
This important role of the substrate is not considered in the mathematical papers of Golle (1953), Finn \& Wilson (1954) and the other authors mentioned above. This has led to some incorrect conclusions. For example, Golle (1953) discusses at some length the mathematics of a series of two or more culture vessels run in cascade, and concludes that there are distinct advantages in this procedure. Now it is apparent from the theory given earlier that over the useful range of flow-rates in a continuous culture, the substrate is nearly completely utilized and the issuing medium virtually exhausted; hence negligible growth could occur in any subsequent culture vessels in series with the first. Our experimental results confirm that this almost complete utilization of substrate does in fact occur.

It is to be emphasized that the theoretical treatment of continuous culture given in this paper is based on the minimum number of extremely simple postulates. It might well be objected that even under ideal conditions the growth behaviour of bacteria cannot be completely represented by such simple equations as (2), (3) and (4). Further refinement of the theory will undoubtedly become necessary as knowledge of the subject increases. The gratifying degree of agreement between experimental and predicted results shows, however, that the basic principles of the theory must be sound. Qualitatively, it provides a good general picture of the behaviour of a continuous culture, and quantitative agreement is good over most of the range. It is necessary to discuss, however, the quantitative deviations from theory found at high flowrates, and in particular the apparent existence of steady states at dilution rates higher than the maximum specific growth rate $\mu_{m}$.

According to equation (27) the specific growth rate must be equal to the dilution rate; when the latter exceeds the maximum possible growth rate, complete 'wash-out' should occur and no steady state should be possible. Since steady states were in fact found with $D>\mu_{m}$, this must mean that either (a) the maximum growth rate in a continuous culture is higher than in a batch culture (the value of $\mu_{m}$ was determined from batch culture experiments), or (b) the wash-out rate is less than would be predicted from equation (1). We believe the latter explanation to be correct.

A possible reason for an organism growing faster in continuous culture than in batch culture might be the selection of faster-growing mutants. However, as previously mentioned, no evidence for any permanent selection of this kind was found, the value of $\mu_{m}$ being unchanged after long periods of continuous culture. Another explanation could be based on the normal variation in the growth-rates (or generation times) of individual bacteria; in a continuous culture the faster-growing bacteria might be selected. This possibility seems to be eliminated by the work of Powell (1955), who found that there is zero correlation between the generation time of a bacterium and the generation times of the two daughter-cells into which it divides. In other words, an unusually fast-growing organism is just as likely as not to have unusually slow-growing progeny; selection would therefore not affect the mean generation time.

The alternative explanation, suggested by our colleague Mr E. O. Powell, is 
that the actual wash-out rate in the culture vessel is effectively less than that given by equation (1). Though this equation has been accepted by all previous writers on the subject, it is based on the unverified assumption of "perfect mixing' within the culture vessel. This implies that each drop of liquid entering the vessel is uniformly distributed throughout its contents in an infinitesimal time, a condition difficult to attain in practice even with vigorous stirring. Incomplete mixing means that the dilution rates in different regions of the culture vessel will not be uniform; there will be a distribution of local dilution rates about the mean or overall dilution rate $\bar{D}=f / v$. Hence when $\bar{D}$ is greater than the critical value $D_{c}$ (calculated from equation 12) there might still be regions within the culture vessel where the local dilution rate was less than $D_{c}$, and in these regions organisms will continue to be produced, so that complete wash-out will not occur. Rough calculations show that quite small deviations from perfect mixing could have surprisingly large effects, owing to the steep descent of the $D$ against $x$ curve in the critical region (Fig. 1). A theoretical and experimental study of this subject is being made by $\mathrm{Mr} \mathrm{E}$. O. Powell, and it will not be further discussed here, but we believe that the greater part of the deviations from theory found in our experiments can be explained in this way. This view is reinforced by the fact that in similar experiments with the same organism in a laboratory scale continuous culture apparatus fitted with a highly efficient mixing system, the deviations from theory at high flow rates are very small (Herbert, D., to be published).

Another 'apparatus effect' can produce results similar to those of imperfect mixing. During long experiments, a solid film of bacteria of considerable thickness builds up on the walls of the culture vessel above the liquid level, portions of which are continually becoming detached by splashes or condensed liquid running down the walls. This continued re-inoculation of the culture from the walls also leads to a continued production of bacteria at flow-rates which should theoretically cause complete wash-out. It is difficult to estimate the magnitude of this inoculation rate, but suppose it to be $w$ g. bacteria/l./hr., then it can be shown that

$$
\tilde{x}=\frac{w}{D-D_{c}} .
$$

Hence if $D$ is only a little above $D_{c}$, a quite small value of $w$ can produce a quite high value of $\tilde{x}$.

Other imperfections in the apparatus (e.g. short-term fluctuations of flowrate or liquid level about the mean values) can also be shown to have effects in the same direction. On the whole, it appears that most of the observed discrepancies can be attributed to 'apparatus effects' of this sort, rather than to inadequacy of the biological side of the theory. Such effects, however, cannot account for the apparent decrease in the yield constant $Y$ at high flow-rates, and it is possible that the independence of yield constant and growth rate assumed in equation (4) may need to be modified; this point is now under investigation.

On the practical side, we consider that the results reported show that the continuous culture of bacteria on a fairly large scale is a readily feasible 
proposition with apparatus of quite simple design. Complexity was deliberately avoided and the apparatus was designed for production rather than research purposes; nevertheless, the degree of control available permits of useful quantitative investigations. Results are comparable with those obtained in smaller and more complicated types of laboratory continuous culture apparatus in use in this Department. The high production rate may be emphasized; though the working capacity of the culture vessel was only $20 \mathrm{l}$., no difficulty was found in producing culture at the rate of $300 \mathrm{l}$./day and higher rates could be achieved with faster-growing organisms. The plant could be scaled up considerably without any major changes in design.

Contamination, often alleged to be a major obstacle to the operation of continuous culture on a plant scale, was not found to be a real difficulty, and runs of several months' duration are now routine. Another alleged difficulty, that of mutation, has not troubled us at all, no changes in the culture having been observed after long periods of continuous operation. In this respect our results are at variance with those of other workers (summarized by Novick, 1955) who have used continuous culture techniques as a means of studying mutation rates. It might be argued that this was due to the type of growth medium used in our experiments, since almost every mutation (of those affecting nutrition at least) would be at a disadvantage compared with the parent type. However, we have had similar experiences with other types of chemically defined and complex growth media, and it could also be argued that in much of the work done on mutations in continuous culture the conditions have been favourable for mutant survival. For a given organism and growth medium, we believe that mutants are somewhat less likely to build up in a continuous culture than in a batch culture. For mutations are rare events arising singly, and a single organism with a generation time $\tau$ has a probability of $1-\mathrm{e}^{-D^{\tau}}$ of being washed out of the culture vessel before it has divided once. Hence an appreciable fraction of the mutants arising in a continuous culture will be removed before they have progeny, while in a batch culture all will remain.

In both the theoretical and experimental parts of this paper, continuous cultivation has been regarded as a process for converting substrate into bacteria - a deliberately one-sided approach. Obviously it has many other aspects; for example, as a research tool for elucidating problems of biosynthesis or as a process for the production of metabolic products. It is hoped to make these the subjects of future investigations.

We are grateful to Dr D. W. Henderson for continued encouragement in this work, to Mr E. O. Powell for invaluable discussions and advice on theoretical aspects and to Mr S. Peto for assistance with statistical problems.

\section{REFERENCES}

Adams, S. L. \& Hungate, R. E. (1950). Continuous fermentation cycle times; prediction from growth curve analysis. Industr. Engng Chem. 42, 1815.

Chain, E. B., Paladino, S., Ugolini, F. \& Callow, D. S. (1954). Pilot plant for fermentation in submerged culture. R.C. Ist. sup. Sanit. 17, 132. 
Cooper, C. M., Fernstrom, G. A. \& Miller, S. A. (1944). Performance of agitated gas-liquid contractors. Industr. Engng Chem. 36, 504.

Danckwerts, P. V. (1954). Continuous flow of materials through processing units. Industr. Chem. Mfr. 30, 102.

Dawson, P. S. S. \& Pirt, S. J. (1954). Engineering and microbiological processes. Chem. \& Ind. (Rev.) p. 282.

Elsworth, R., Telling, R. C. \& Ford, J. W. S. (1955). Sterilization of bacteria by heat. J. Hyg., Camb. 55, 445.

Finn, R. K. \& Wilson, R. E. (1954). Population dynamics of a continuous propagator for micro-organisms. Agric. Fd. Chem. $2,66$.

Golle, H. A. (1953). Theoretical considerations of a continuous culture system. Agric. Fd. Chem. 1, 789.

Mrles, A. A. \& Misra, S. S. (1938). The estimation of the bactericidal power of the blood. J. Hyg., Camb. 38, 732.

Monop, J. (1942). Recherces sur la croissance des cultures bactériennes. Paris: Hermann \& Cie. :

Monod, J. (1950). La technique de culture continue; théorie et applications. Ann. Inst. Pasteur. 79, 390.

Neish, A. C. (1952). Analytical Methods for Bacterial Fermentations. Nat. Res. Coun. Canada, Rep. no. 46-8-3 (2nd revision), Saskatoon.

Northrop, J. H. (1954). Apparatus for maintaining bacterial cultures in the steady state. J. gen. Physiol. 38, 105.

Novick, A. (1955). Growth of bacteria. Annu. Rev. Microbiol. 9, 97.

Novick, A. \& Szimard, L. (1950). Experiments with the Chemostat on spontaneous mutations of bacteria. Proc. nat. Acad. Sci., Wash. 36, 708.

Powell, E. O. (1955). Some features of the generation times of individual bacteria. Biometrika, 42, 16.

Warner, F. E., Cook, A. M. \& Train, D. (1954a). Engineering and microbiological processes. Chem. \& Ind. (Rev.) p. 114.

Warner, F. E., Cook, A. M. \& Train, D. (1954b). Engineering and microbiological processes. Chem. \& Ind. (Rev.) p. 283.

(Received 15 November 1955) 